\title{
Hierarchically Structured Neural Networks for Printed Hangul Character Recognition
}

\author{
Sung-Bae Cho and Jin H. Kim \\ Computer Science Department \\ Korea Advanced Institute of Science and Technology \\ P.O. Box 150, Cheongryang, Seoul 130-650, Korea \\ sbcho@csd.kaist.ac.kr (Internet)
}

\begin{abstract}
In this paper, we propose a hierarchical newral network which practically recognizes printed Hangul(Korean) characters. This system is composed of a type classification network and six recognition networks. The former classifies input character images into one of the six types by their overall structure, and the latter further classify them into character code. Furthermore, a training scheme including systematic noises is introduced for the improving the generalization capabilities of the networks. Experiments are conducted with most frequently used 990 printed Hangul characters. By the noise included training, the recognition rate amounts up to $98.28 \%$, which is superior to the conventional backpropagation network. After all, neural network approach turns out to be very reasonable through a comparison with statistical classifer and an analysis of generalization capability.
\end{abstract}

\section{Introduction}

Recently, neural netwoks have been spotlighted in the various fields of sciences. The attractiveness of non-programmed adaptability has encouraged many researchers to apply neural network to their problems $[4,10]$. It has been reported that neural networks are adequate for pattern recognition problems, but the number of classes used for experiments was too small to show the capabilities for the real world problems. We have chosen to base our experiments on a printed Hangul character recognition, whose difficulties can be measured by the large set of classes and massive interconnections. Since larger networks require increasing amounts of training time and patterns, the computational complexity of the learning process quickly reaches unmanageable proportions [11].

In this paper, designed and implemented are neural networks for printed Hangul character recognition with the input data obtained from the optical scanner. Even for the printed character, building a useful Hangul recognition system is not simple because more than thousands of characters are used for every day life, and characters are very similar as well as have noises and deformations due to the sensor operation. In order to overcome these difficulties, the system is hierarchically organized. The hierarchical classification scheme involves two different networks, each of which is designed for a specific task. It is composed of a type classification network and six recognition networks. The former roughly classifies input character images into one of the six types by their overall structure, and the latter further classify them into character code.

Since for real world tasks the underlying training set contains only a small number of characters, we propose a training scheme including systematic noises which is useful for large scale problems. The noise included training scheme adds noises systematically to the given training patterns. This results in the same effect as expanding the number of training patterns and, therefore, improves its generalization capability.

The rest of this paper is organized as follows. In Chapter II, we propose the hierarchical neural networks for recognizing printed Hangul characters. A new training scheme including systematic noises is explained in Chapter III, and experimental results with most frequently used 990 Hangul characters are presented in Chapter IV. Finally, conclusions and further researches are discussed in Chapter V.

\section{Hierarchical Neural Networks}




\subsection{Structure of Hangul Characters}

Unlike the Chinese written language, Hangul character is phonetic in nature. Hangul characters, on the other hand, do not have the linear structure of words in western languages. They are formed roughly in square which is characteristic of Chinese characters. A Hangul character is produced by combining two or four alphabets among the 14 consonants and 10 vowels. The number of distinct characters which can be formed from the 24 basic alphabets is 11,172 and about 2,300 of them are used for daily life.

Rather than forming words, as English and other western languages do, Hangul letters form two dimensional clusters called characters. Each character represents a syllable. A word usually consists of between one and four syllables. A character cluster may be composed of a head consonant, a vowel and zero to two bottom consonants. A general structure of Hangul character is presented in Figure 1 where V1 means the vertical vowel, V2 means the horizontal vowel, $\mathrm{C} 1$ means the head consonant and $\mathrm{C} 2$ means the bottom consonant. According to the shape of the vowel included in the character and the existence of the bottom consonant, the types of Hangul characters can be divided into the six groups. In Figure 2, the structure of six types are shown.

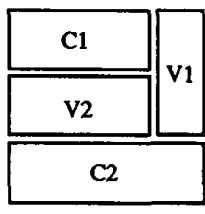

Figure 1. General Structure of Hangul Characters

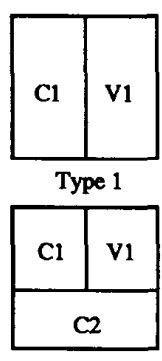

Type 4

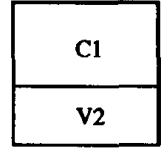

Type 2

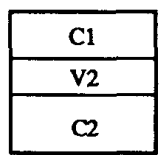

Type 5

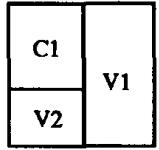

Type 3

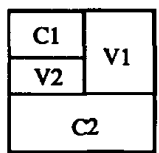

Type 6

Figure 2. Six Types of Hangul Characters

\subsection{Overall Structure of Recognition Networks}

At the beginning of research, we implemented a neural network using a simple backpropagation network with a single hidden layer which has $1,600(40 \times 40)$ input nodes, 40 hidden nodes and 10 output nodes which are distributively encoded. This network, however, did not converge when the number of classes of training samples exceeded one hundred characters. To overcome this obstacle, it is necessary to recognize each alphabet by alphabet not character by character on the ground of divid and conquer strategy.

So are designed hierarchical networks, which are composed of a type classification network and six recognition networks. The former roughly classifies input character images into one of the six types by their overall structure, and the latter further classify them into character code. Figure 3 and Figure 4 shows the overview of the proposed system. The idea of customized receptive field for each recognition network is based on the observation that every character in the same type has the structural similarity.

Hierarchical networks have the following advantages over a simple backpropagation network; higher recognition performance is obtained, and the training time is shortened. In addition to these merits, the system is organized modularly, and simply adds a new group if new character set is required to be trained.

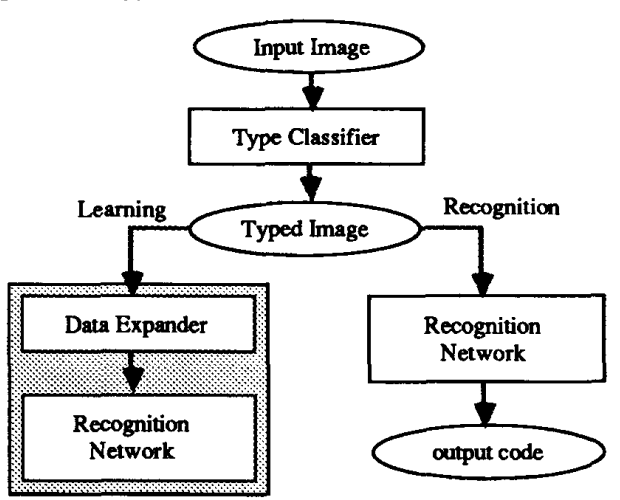

Figure 3. Overview of Hangul Character Recognition System

\subsubsection{Type Classification Network}

Since Hangul characters can be grouped six types, the recognition system is greatly simplified if the type 
of input image is known a priori. First of all, our system determines the type of input image and activates the corresponding network for real character recognition. Figure 5 shows the structure of type classification network.

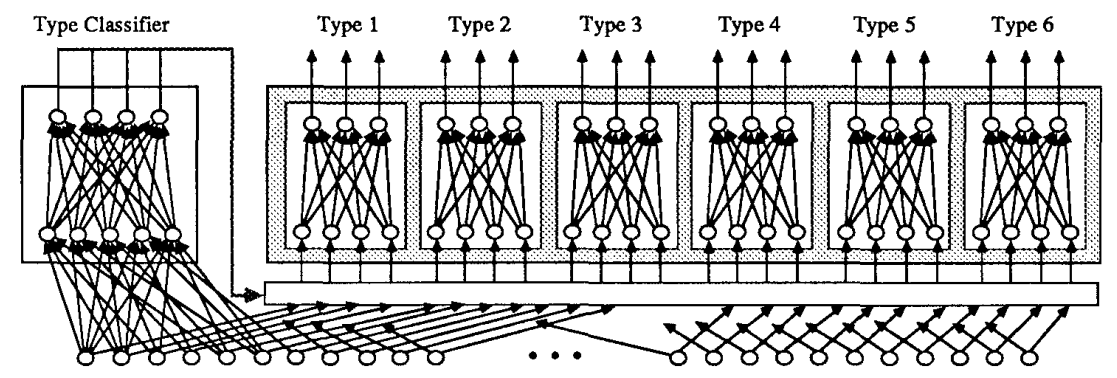

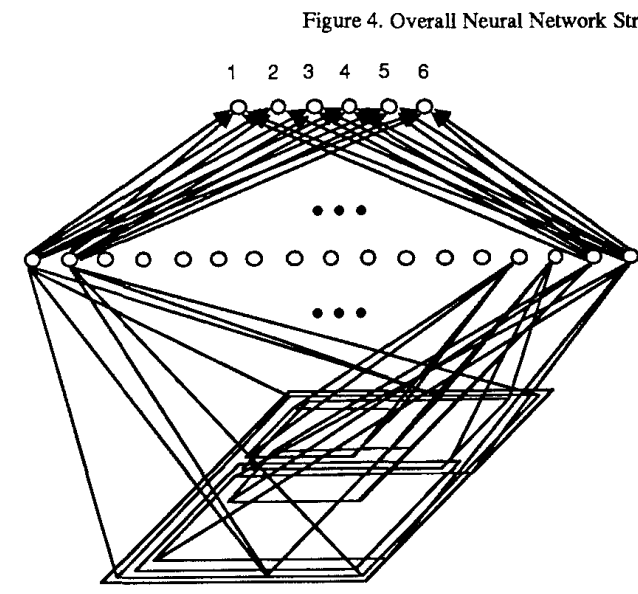

Figure 5. Type Classification Neural Network

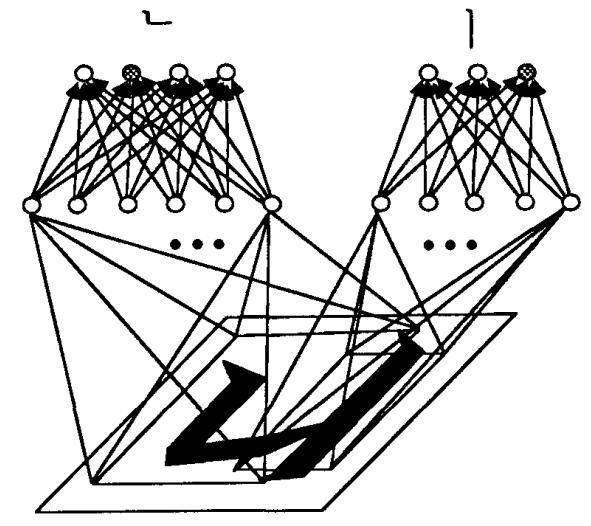

Figure 6. Neural Network for Typed Character Recognition (A Case of Type 1)

Type classifier is a partially connected backpropagation network which has 1,600 input nodes, 17 hidden nodes and 6 output nodes. Each node of hidden layers is connected to the specific input area which represents the possible alphabet. The first node, for example, is connected to the consonant area of type 1. Each hidden node arrives at a local decision, and output nodes summarizes and reaches the final decision for type determination. This structure can be justified by the observation that each character plays an important role for classifying the type. In fact, the number of interconncetions of this network is 5,915 , while a fully connected network requires $27,302(40 \times 40 \times 17+17 \times 6)$.

\subsubsection{Recognition Networks}

Now, it is possible to recognize the input character as alphabet by alphabet because every character of the same type has its alphabets in a nearly unique position. Figure 6 shows the structure of recognition network for the character of the first type. Each recognition network is structured for recognizing its alphabet, and has a single hidden layer which has 40 nodes. It has been proved that a multi-layer perceptron with at most two hidden layers can form any arbitrarily complex decision regions in a feature space which is the ultimate goal of any classifier [7].

\section{Noise Included Training}

A large scale problem usually has the large variances of input data, and this results in the bad 
generalization from a necessarily limited set of training data. It is the major obstacle for achieving a high recognition performance. Researchers have tried several different approaches to overcome the geometrical variations of input data $[3,6,9]$. Since for real world problems, the underlying training set contains only a small number of data, an automatic expansion of the training set improves the learning capabilities of the network. For speech recognition problems, it was reported that recognition performance was improved by introducing the random or the temporal distortions into the training data [1,8]. In this Chapter, proposed is a new distortion technique to generate some new training data from the given data which is useful in general problem domains.

From the given training data, additional modified patterns are derived by shifting them over a predetermined set of directions, such as up, down, left and right. This method expands the number of training patterns, and what is more, its artificial expansion is performed optimally. After all, the noise included training scheme adds noises systematically to the given training patterns. This results in the same effect as expanding the number of training patterns and, therefore, improves its generalization capability. This leads to networks which can respond in a more flexible way to variations in the input data.

\section{Experimental Results}

\subsection{Environments for Experiments}

Experiments are conducted with most frequently used 990 printed Hangul characters on the Cray2s super computer. We used the laser printer output through the Microtek MSF300C scanner for the experiment. After analyzing the horizontal projections and the vertical projections, a maximum $40 \times 40$ binary image of each character is extracted from the scanned document. Figure 7 shows the real example of scanned characters.

\subsection{Analysis of Results}

The recognition results of conventional and noise included learning method are shown in Tabel 1 and Table 2 respectively. By the noise included learning, the recognition rate amounts up to $98.28 \%$, which is superior to the conventional backpropagation learning method's $95.45 \%$. Figure 8 compares

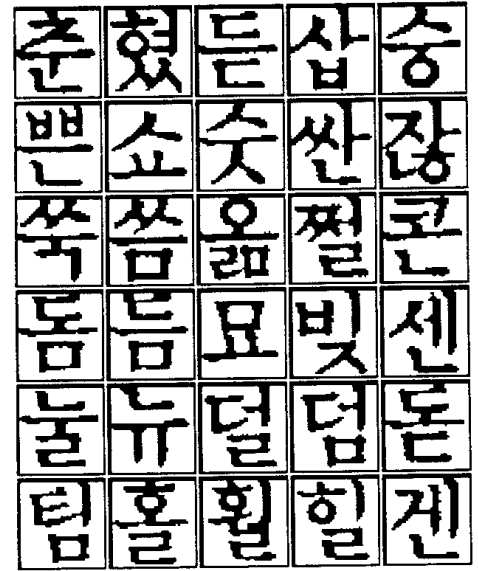

Figure 7. Practical Example of Scanned Image both of these recognition rates according to the type.

Figure 9 shows a comparison with tree classifier which is the efficient implementation of nearest neighbor rule [2], while TC means tree classifier and NN means neural network. This comparison proves the fact that multi-layer perceptron classifiers trained with the backpropagation algorithm can perform as well as and sometimes better than conventional classifiers [5].

For examining the generalization capability of proposed neural netowk, we tried to recognize the 2,350 characters using the system trained with 990 characters. Table 3 shows the recognition results, which is acceptable.

\section{Concluding Remarks}

In this paper, we propose the hierarchical neural networks for recognizing large set characters and a training scheme included systematic noises which is useful for applying neural network to real world problems, and experiments with most frequently used 990 Hangul characters are conducted for showing the usefulness of the proposed.

Further works are the modification of proposed networks for recognizing the multi-font, multi-size characters, and the combination of neural network approach with conventional recognition methods for better performances. 


\section{References}

[1] R. K. Bernhard and A. K. Wolfgang, "Design of Hierarchical Perceptron Structures and their Application to the Task of Isolated-word Recognition," in Proceedings of the IEEE IJCNN, Vol. I, pp. 243-249, 1989.

[2] R. O. Duda and P. E. Hart, Pattern Classification and Scene Analysis, New York, Wiley Press, 1973.

[3] K. Fukushima, "A Neural Network for Visual Pattern Recognition," IEEE Computer, Vol. 21, No. 3, March 1988.

[4] R. Hecht-Nielsen, "Neurocomputer Applications," NATO ASI Series F: Computer and Systems Sciences, Vol. 41, pp. 445-453, 1988.

[5] W. Y. Huang and R. P. Lippmann, "Comparisons between Neural Net and Conventional Classifiers," in Proceedings of the IEEE ICNN, Vol. IV, pp. 485-493, 1987.

[6] A. Khotanzad and J. H. Lu, "Distortion Invariant Character Recognition by a Multi-Layer Perceptron and Backpropagation Learning," in Proceedings of the IEEE ICNN, Vol. I, pp. 625-632, 1988.

[7] R. O. Lippmann, "An Introduction to Computing with Neural Nets," IEEE ASSP Magazine, pp. 4-22, April 1987.

[8] T. Matsuoka, H. Hamada and R. Nakatsu, "Syllable Recognition Using Integrated Neural Networks," in Proceedings of the IEEE IJCNN, Vol. I, pp. 251-258, 1989.

[9] W. L. Reber, "An Artificial Neural System Design for Rotation and Scale Invariant Pattern Recognition," in Proceedings of the IEEE ICNN, Vol. IV, pp. 277-283, 1987.

[10] D. E. Rumelhart and J. L. McClelland, Parallel Distributed Processing : Explorations in the Microstructure of Cognition, MIT Press, 1986.

[11] A. Waibel, "Connectionist Glue: Modular Design of Neural Speech Systems," in Proceedings of the 1988 Connectionist Models Summer School, pp. 417-425, 1988.

\begin{tabular}{|c|c|c|c|c|c|c|c|}
\hline \multicolumn{2}{|c|}{} & \multicolumn{2}{|c|}{$\begin{array}{c}\text { Group } \\
\text { Identitication }\end{array}$} & \multicolumn{2}{c|}{$\begin{array}{c}\text { Character } \\
\text { Recognition } \\
\text { in each Grour }\end{array}$} & \multicolumn{2}{c|}{ Overall } \\
\hline \hline Group & \#char & \#error & $\begin{array}{c}\% \\
\text { correct }\end{array}$ & \#error & $\begin{array}{c}\% \\
\text { correct }\end{array}$ & \#error & $\begin{array}{c}\% \\
\text { correct }\end{array}$ \\
\hline \hline 1 & 114 & 0 & 100.00 & 0 & 100.00 & 0 & 100.00 \\
\hline 2 & 68 & 3 & 95.59 & 2 & 97.06 & 5 & 92.65 \\
\hline 3 & 42 & 0 & 100.00 & 6 & 85.71 & 6 & 85.71 \\
\hline 4 & 480 & 1 & 99.79 & 16 & 96.67 & 16 & 96.67 \\
\hline 5 & 251 & 0 & 100.00 & 15 & 94.02 & 15 & 94.02 \\
\hline 6 & 35 & 3 & 91.43 & 0 & 100.00 & 3 & 91.43 \\
\hline \hline Overall & 990 & 7 & 99.29 & 39 & 96.06 & 45 & 95.45 \\
\hline
\end{tabular}

Table 1. Recognition Rate of Conventional Learning

\begin{tabular}{|c|c|c|c|c|c|c|c|}
\hline & \multicolumn{2}{|c|}{$\begin{array}{c}\text { Group } \\
\text { Identification }\end{array}$} & \multicolumn{2}{c|}{$\begin{array}{c}\text { Character } \\
\text { Recognition } \\
\text { in each Grour }\end{array}$} & \multicolumn{2}{c|}{ Overall } \\
\hline \hline Group & \#char & \#error & $\begin{array}{c}\% \\
\text { correct }\end{array}$ & \#error & $\begin{array}{c}\% \\
\text { correct }\end{array}$ & \#error & $\begin{array}{c}\% \\
\text { correct }\end{array}$ \\
\hline \hline 1 & 114 & 0 & 100.00 & 0 & 100.00 & 0 & 100.00 \\
\hline 2 & 68 & 3 & 95.59 & 0 & 100.00 & 3 & 95.59 \\
\hline 3 & 42 & 0 & 100.00 & 2 & 95.24 & 2 & 95.24 \\
\hline 4 & 480 & 1 & 99.79 & 7 & 98.54 & 7 & 98.54 \\
\hline 5 & 251 & 0 & 100.00 & 2 & 99.20 & 2 & 99.20 \\
\hline 6 & 35 & 3 & 91.43 & 0 & 100.00 & 3 & 91.43 \\
\hline \hline Overall & 990 & 7 & 99.29 & 11 & 98.89 & 17 & 98.28 \\
\hline
\end{tabular}

Table 2. Recognition Rate of Noise Included Learning 


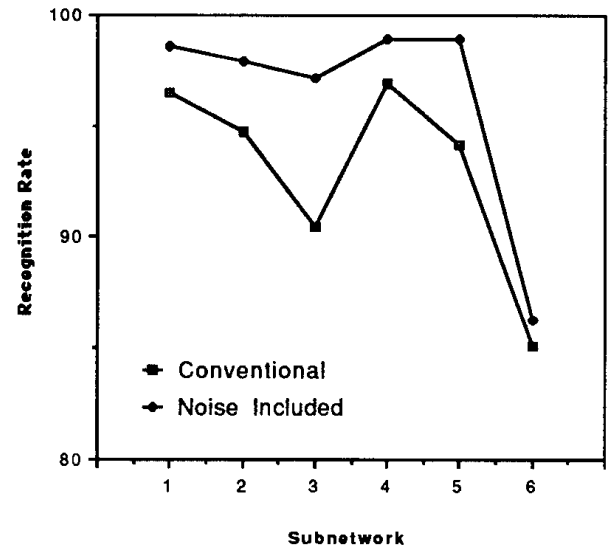

Figure 8. Comparison of Conventional vs Noise Included Learning

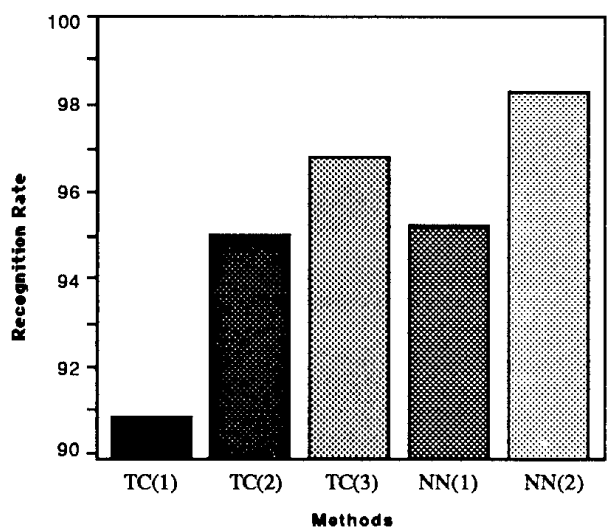

Figure 9. Comparison of Neural Network with Statistical Method

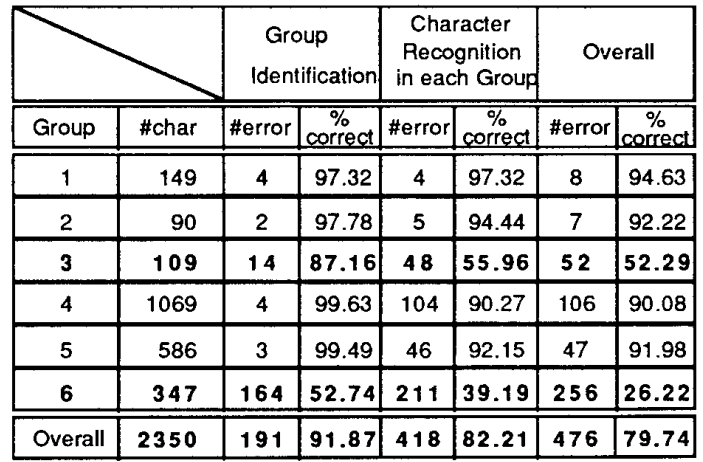

Table 3. Generalization Capability : Recognition Rate of 2,350 Characters 